\title{
The Efficacy of Biomechanical Training in Decreasing Work- Related Injuries in a Shipping/Receiving Company: A Comparison Study
}

\section{Gayle R*, Curry A and Carley P \\ Doctorate of Physical Therapy, Department at American International College, USA}

*Corresponding author: Rachelle Gayle, Doctoral student, Doctorate of Physical Therapy Department at American International College, 1000 State Street, Springfield MA. 01109, USA, Email: Rachelle.Gayle@aic.edu

\section{Research Article \\ Volume 2 Issue 2}

Received Date: April 25, 2018

Published Date: April 30, 2018

DOI: $10.23880 /$ eoij-16000144

\section{Abstract}

Background of Study: The shipping industry has become progressively more mechanized in the form of conveyor belts, sorting machines, and scanning devices but not all jobs can be automated. It is common for shipping industry's employees to work 8-12 hours shifts performing various lifting tasks, machine maintenance, and manipulating awkward equipment. Based on the Occupational Safety and Health Administration (OSHA) reports, there are many shipping and receiving work-related injuries involving mainly the shoulder, back, neck, and feet areas. Many of work tasks involve repetitive actions that include lifting and equipment operation. Even though workers are trained in lifting techniques, there are still frequent injuries reported in this setting causing both employee safety and employer financial concerns.

Objective of Study: The purpose of this comparison study is to reduce the prevalence of work related injuries in the shipping and receiving industry through educating employees in proper biomechanics to perform their daily job safely.

Design of the Study: A pre-survey was initially administered. Then followed by a 30-minute presentation for education. A second post survey was then provided for comparison. OSHA injury reports were reviewed a year later following the educational sessions.

Methods: Pre and post anonymous surveys were completed by94 employees in various positions within a shipping/receiving company. The survey consisted of both multiple choice and circle all that apply. The survey was designed to assess: knowledge, activity level, and interest regarding biomechanics. The training provided by the investigators consisted of visual, verbal, and demonstrative techniques for proper biomechanical posturing. This included lifting, pushing, pulling, standing, twisting, and sitting, as well as basic stretching techniques to be included in daily routines going forward.

Results: The results of the comparison between pre-education and post-educational sessions show an approximate $25 \%$ increase in employee appreciation and an understanding of posture, stretching, and regular exercise as is relates to daily 


\section{Ergonomics International Journal}

life and their work place. Following the educational sessions in the reported fiscal year of 2016-2017 the OSHA reported injuries for lifting decreased by $66 \%$ and the injuries involving equipment handling decreased by $90 \%$.

Limitations: The limitations of this study include: small sample size, limited amount of time for educational sessions and time constraints of data collection due to shipping companies operational window, some employees had received some type of physical therapy in the past, and the OSHA data that has been collected regarding the injuries reported from the shipping company are not yet complete due to fiscal year ending in September 2018.

Conclusion: Based on the subjective data collected via survey and the decrease in OSHA reported work-related injuries the authors conclude that with proper education and awareness regarding stretching, exercise, and proper biomechanics it is possible to decrease the number of work-related injuries within the shipping and receiving industry.

\section{Introduction}

In the current climate of an ever-changing technologically based industrial society, change and advancement have become the norm throughout the global workforce. While business booms and technology advances, the human body stays relatively the same; which means: repetitive motion and faulty biomechanics result in injury. Currently, one of the greatest revolutions in the American culture is mail order products. For the consumer, the ability to have a variety of commodities delivered to their doorstep is a great convenience. The problem lies in how the commodities make it from point $\mathrm{A}$ to point $B$, through the shipping and receiving industry, one place where automation has not yet completely taken over.

The shipping industry has become progressively more mechanized in the form of conveyor belts, sorting machines and scanning devices but not all jobs can be automated. It is common for shipping industry's employees to work 8-12 hours shifts performing various lifting tasks, machine maintenance, and manipulating awkward equipment. Based on the Occupational Safety and Health Administration (OSHA) reports, there are many shipping and receiving work-related injuries involving mainly the shoulder, back, neck, and feet areas. Many of work tasks involve repetitive actions that include lifting and equipment operation. Even though workers are trained in lifting techniques, there are still frequent injuries reported in this setting causing both employee safety and employer financial concerns.

According to Occupational Safety and Health Administration:

In addition to their social costs, workplace injuries and illnesses have a major impact on an employer's bottom line. It has been estimated that employers pay almost $\$ 1$ billion per week for direct workers' compensation costs alone. The costs of workplace injuries and illnesses include direct and indirect costs. Direct costs include workers' compensation payments, medical expenses, and costs for legal services. Examples of indirect costs include training replacement employees, accident investigation and implementation of corrective measures, lost productivity, repairs of damaged equipment and property, and costs associated with lower employee morale and absenteeism (www.osha.gov).

Due to the significant personal, social, and financial impacts of injury, there is an increased need for employee education in biomechanics to decrease the risk of injury. The number of OSHA reported injuries can be reduced through education, biomechanical training, and instruction in daily stretches for the shipping and receiving industry workers. The purpose of this comparison study is to reduce the prevalence of work related injuries in the shipping and receiving industry through educating employees in proper biomechanics to perform their daily job safely. The objective is to determine if with proper education in biomechanics and a daily stretching routine can the OSHA reported number of workplace injuries be reduced in a local shipping and receiving company. The shipping and receiving company which was surveyed has requested to remain nameless and will only be referred to as a shipping company herein.

There is substantial research suggesting that with a fundamental knowledge in basic anatomy and proper body mechanics, work related and repetitive motion injuries can be significantly reduced. With appropriate education and biomechanical training, employees work behaviors changed resulting in decreased risk for injuries and consequently transforming the work place atmosphere [1]. Work related arm, neck and shoulder pain/injury causing absenteeism results in costs that 


\section{Ergonomics International Journal}

range between $\$ 45-54$ billion in the United States annually [2]. Research indicates that regular strength training for individuals working in industrial environments can reduce upper extremity pain [3]. In a self-reported questionnaire study, the objective was to identify what work related factors were precipitating work related injuries. It was found that with the 3,361 respondents there was a 39\% reduction in neck/shoulder pain, a $47 \%$ reduction in low-back pain, and a $51 \%$ reduction in elbow/hand region pain following education [4]. Ultimately, ergonomic investigators are finding that with proper training, and improvement in workplace organization, employee attitudes are improved and musculoskeletal injuries decrease [5]. One group of investigators are working to devise a protocol for Mason's to decrease the high rate of musculoskeletal injuries that occur frequently throughout the trade. Their research has found that with education and demonstrative techniques, injuries can be significantly decreased [6]. When evaluating ergonomic programs, Driessen et al. discovered that in cases where there were not decreased prevalence of neck and low back injuries, the ergonomic protocols that were put into place were not implemented correctly indicating the need for and importance of, proper ergonomic training for workers [7].

\section{Methods}

A survey was administered anonymously to a total of 94 employees, before and after a 30-minute training session presented by the investigators: two student doctors of physical therapy. The participants were not randomly selected. They participated based on availability to attend an educational seminar during a workday. None of the surveys collected were excluded. Each participant was given a random number as they entered the room where education was to be provided, and that corresponded as their survey number. The investigators were blinded to the random numbers of the survey. The survey consisted of both multiple choice and circle all that apply questions. The survey was designed to assess: knowledge, activity level, and interest regarding biomechanics. The jobs held by the employees included but were not limited to: conveyor belt monitors, office employees, package delivery, and customer service.

The survey consisted of ten questions. Six of the questions were designed to measure activity, interest, and knowledge of those being surveyed. The other four questions were created to look at experience with injury. Questions one and two read: Do you think that stretching daily is important? Do you stretch regularly? The answer options for both consist of three choices: yes, no, indifferent. These questions inform the surveyors of the initial level of interest and activity regarding stretching. Following the educational sessions, it could be determined if their lesson had an impact on the employees' level of enthusiasm regarding stretching. The same was done for daily exercise in questions three and four. Questions five and six are regarding posture. They read as: Do you think that your posture is important? Do you think that your posture has an influence on your performance at work? With the same options to answer: yes, no, or indifferent. These questions were designed to inform the surveyors whether the employees had knowledge of good biomechanics and if they felt they were important prior to the educational session. Following the education, it could be determined if the session had an impact on the employees' awareness and the level of importance they placed on biomechanics.

The final four questions of the survey addressed pain. Where and when the employee experiences pain, if they have ever missed work due to a work-related injury, and whether they have had physical therapy in the past. The assumption being that if they have had physical therapy in the past, there may be a correlation between those that had answered yes in the first survey to questions one through six and stated they had experience with physical therapy.

The educational session consisted of a review of proper biomechanical posturing with lifting, pushing, pulling, standing, twisting, and sitting. As well as, a review of basic stretching techniques to be included in daily routines and was given to the participants in handouts. The stretches that were included in the daily routine were: gastroc/soleus, hamstring, upper trapezius, levator scapulae, pectoralis major/minor, piriformis, and the common yoga position known as "child's pose." These stretches were chosen because they target the most frequently overused and injured areas of the body. The educators prepared a PowerPoint presentation which focused on individual jobs that they witnessed in the shipping warehouses during observational sessions, as well as concepts in basic anatomy, posture, and bodymechanics. Included with the presentation was a demonstration of proper and improper methods of equipment use. After the survey data was collected and collated into a spreadsheet, the OSHA public records of the shipping company were collected. After one year of delivering the educational sessions and collecting the surveys, the most recent OSHA reports of the shipping company regarding the same injuries were collected again and compared to the initial reports. 


\section{Results}

The results of the comparison between pre-education and post-educational session show an approximate $24.4 \%$ increase in employee appreciation for, and an understanding of posture, stretching, and regular exercise. The OSHA reported injuries for lifting decreased by $66.6 \%$ in the reporting year following the educational sessions and injuries involving equipment handling decreased by $89.9 \%$ (Figure 1).

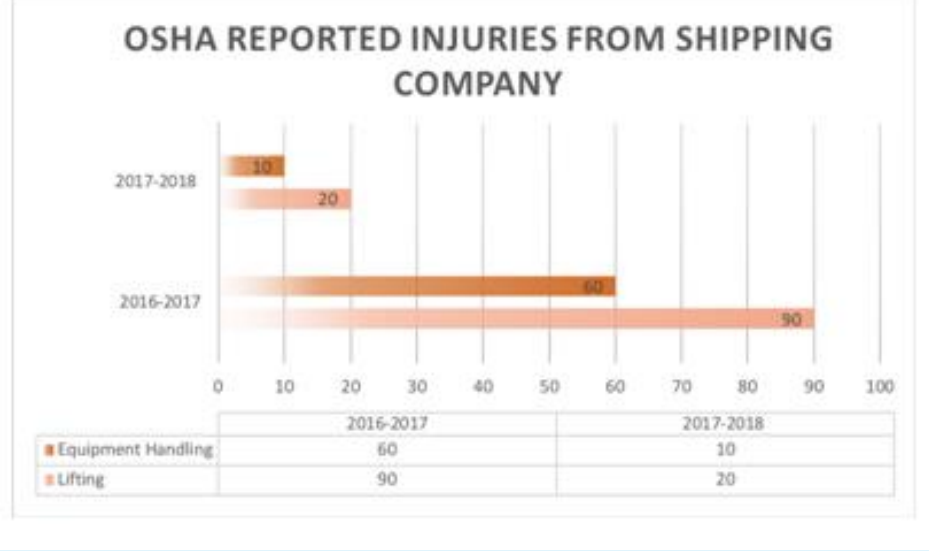

Figure 1: Disclosure: the numbers from the OSHA reports have been rounded in such a way to not disturb the percent difference but to prevent them from being identifiers of the anonymous entity where research was completed.

In the 2016-2017 reporting year for the shipping company there were 60 injuries reported to OSHA that involved equipment handling and 90 injuries reported to OSHA involving lifting. In the next, and current reporting year from 2017-2018 (present), there have been 20 reported lifting injuries a $66 \%$ decrease, and 10 reported injuries related to equipment handling, a $90 \%$ decrease from the previous year.

Initially $54.2 \%$ of those surveyed answered that they think stretching is important prior to the educational session and $79.7 \%$ after the education session. This means that $25.5 \%$ of total participants changed their answers following the session to say that they now believed stretching to be an important activity. It should be noted that $90.1 \%$ of the participants that answered "yes" to the question asking if stretching was important have received some sort of physical therapy in their history. This indicates that they would have been educated on the importance of muscle stretching and exercise. Therefore, their answers may have been different had they not had previous experience with physical therapy (Figure 2).

\section{IS DAILY STRETCHING IMPORTANT?}

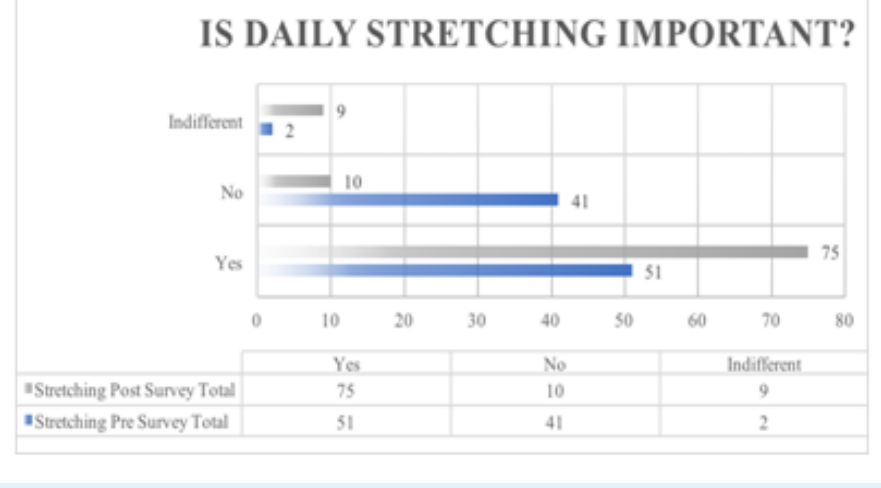

Figure 2: Initially 54\% of those surveyed answered that they think stretching is important prior to educational session and $80 \%$ after education which means that $26 \%$ of total participants changed their answers following education to say that they now believed stretching to be an important activity. 
Based on the responses to the questions regarding exercise, $88.2 \%$ of participants answered "yes" that they believed daily exercise was important in the pre- survey. $24.09 \%$ of those that answered yes reported that they currently exercise 1-2 times per week for at least 30 minutes. $67.4 \%$ reported that they exercise 3 or more times per week for at least 30 minutes. The percentage of post-survey "yes" answers regarding exercise increased to $95.7 \%$ of participants. These findings suggest that while many of the shipping company's employees value exercise as an important part of well-being, they do not all engage in exercise activity in their own lives (Figure 3).

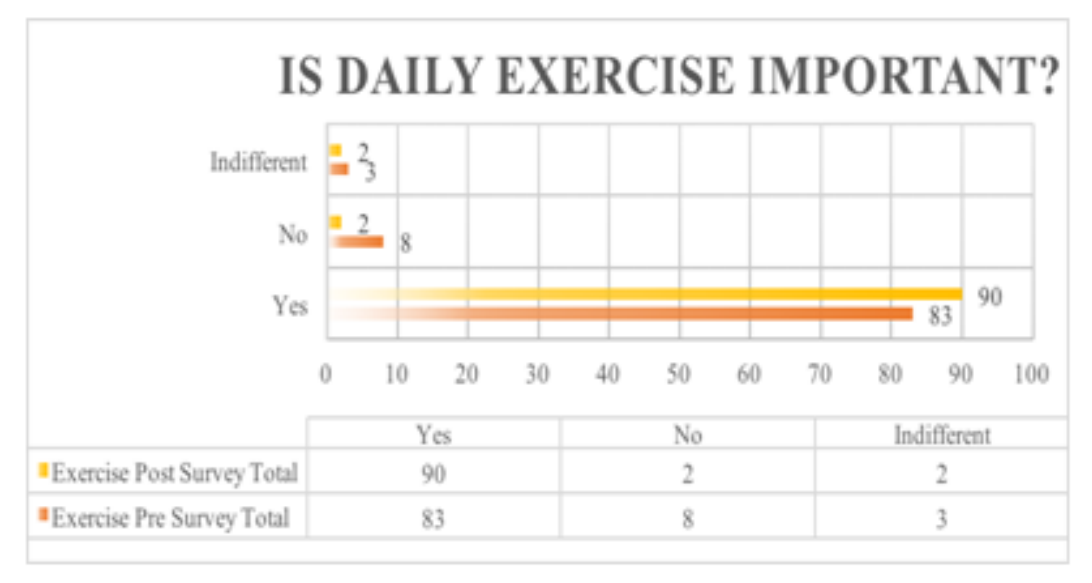

Figure 3: 88\% of participants answered "yes" that they believed daily exercise was important in the pre- survey. The percentage of post-survey "yes" answers regarding exercise increased to $96 \%$ of participants.

In the pre-survey $21.2 \%$ of participants reported that they did not believe posture was important in their job performance. $76.5 \%$ initially said that they did believe that posture was important and that it could affect their job performance. Following the educational session $92.5 \%$ of all participants reported that posture was important and could have an impact on their job performance; an increase of $16.0 \%$ (Figure 4).

\section{IS POSTURE IMPORTANT?}

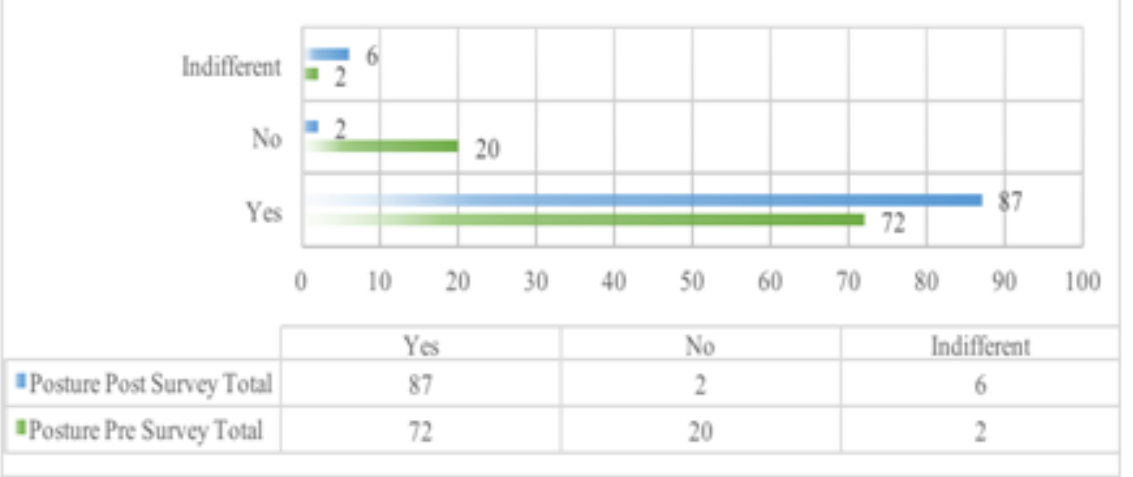

Figure 4: In the pre-survey $21 \%$ of participants reported that they did not believe posture was important in their job performance. $71 \%$ initially said that they did believe that posture was important and that it could affect their job performance. Following the educational session $93 \%$ of all participants reported that posture was important and could have an impact on their job performance; an increase of $22 \%$. 
Questions seven and eight of the shipping employee survey ask where the participant has pain and when the pain occurs. For the question: where do you have pain (Figure 5), two-hundred and six answers were recorded, indicating that respondents selected more than one answer. $34.4 \%$ of responses indicate that the most frequent location of pain is the neck and back, $29.6 \%$ in ankle and feet, $22.3 \%$ in the shoulder, and $13.5 \%$ in the wrist and hand. The same occurred with the question: when does pain occur (Figure 6). There were one hundred and sixty-five responses recorded. 36.9\% during work, $44.8 \%$ after work, $15.1 \%$ on days off, and $3.03 \%$ never.

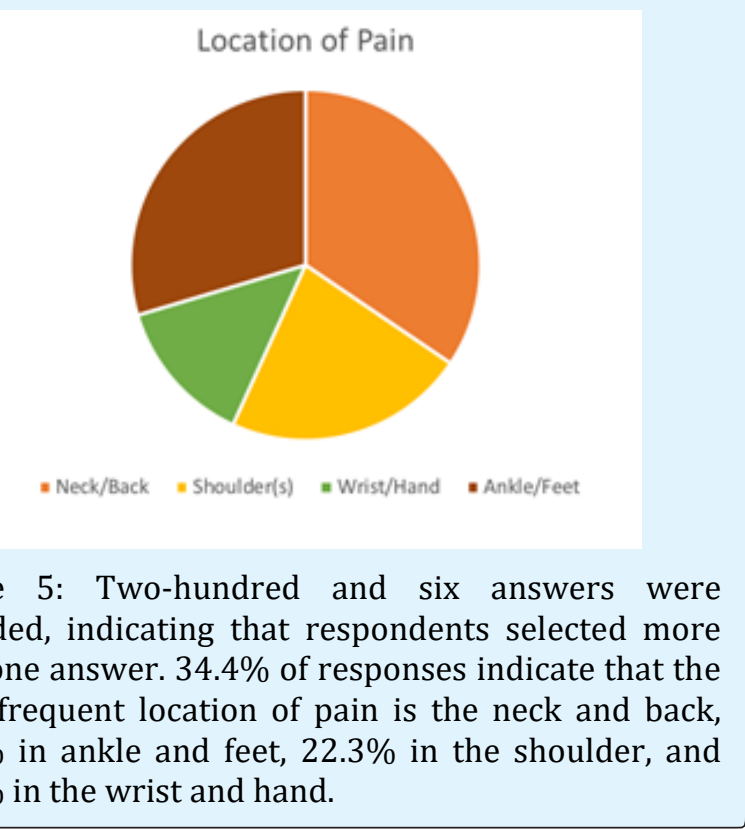

Figure 5: Two-hundred and six answers were recorded, indicating that respondents selected more than one answer. $34.4 \%$ of responses indicate that the most frequent location of pain is the neck and back, $29.6 \%$ in ankle and feet, $22.3 \%$ in the shoulder, and $13.5 \%$ in the wrist and hand.

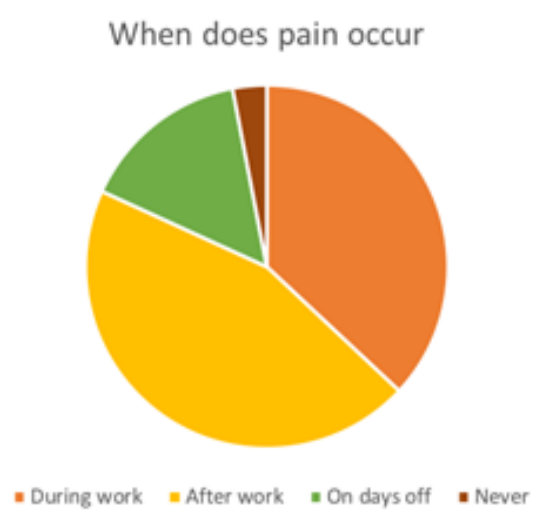

Figure 6: There were one hundred and sixty-five responses recorded. $36.9 \%$ during work, $44.8 \%$ after work, $15.1 \%$ on days off, and $3.03 \%$ never.
In the 2016-2017 reporting year, the shipping company had 60 injuries reported to OSHA that involved equipment handling and 90 injuries reported to OSHA involving lifting. The educational sessions were delivered 2016. In the next, and current reporting year from 2017 2018 (present), there have been 20 reported lifting injuries. This resulted in a $77.7 \%$ decrease. There were 10 reported injuries related to equipment handling, an $83.3 \%$ decrease from the previous year (Figure 1).

\section{Discussion}

Based on the subjective data collected via survey and the decrease in OSHA reported work-related injuries the authors conclude that with proper education and awareness regarding stretching, exercise, and proper biomechanics it is possible to decrease the number of work-related injuries within the shipping and receiving industry. The results of this study imply that with the knowledge and education these employees gained from the educational session they attended; they became more aware of both standing and sitting posture throughout their workday. The employees are now able to properly approach the act of lifting or manipulating an awkward or heavy object. Education and demonstration of safe work duties delivered by two student doctors of physical therapy for 30 minutes was shown to decrease the number of lifting and equipment handling injuries in a small workforce of shipping employees.

This study benefits the profession of physical therapy as it brings the practice out of the clinics and directly into the workplace. This opens the door to physical therapy professionals to find where musculoskeletal injuries are occurring and to begin training individuals in the tasks that are detrimental to human biomechanics. Further research should be conducted to confirm that education/demonstration sessions alone benefit shipping/receiving employees to prevent or reduce workrelated injuries. Research should include follow up over a longer duration of time elapsed between data collected to conclude that initial awareness of proper body mechanics is being upheld throughout the work day.

The limitations of this study include: the small sample size in comparison with the amount of people that work in the shipping and receiving industry nationwide, the lack of time between surveys given, as they were given prior to the educational session, then at the end of the session, which could have influenced their answers. Had there been a greater length of time between surveys, there may have been more accurate results. Another limitation is that a large percentage of surveyed 


\section{Ergonomics International Journal}

employees had received some type of physical therapy in the past and therefore may have already had some sort of base knowledge and practice of proper postural, stretching, and exercising techniques. Also, the OSHA data that was collected regarding the injuries reported from the shipping company are not yet complete as the reporting term ends in September 2018. The OSHA numbers used in this study were collected prior to the educational sessions the authors provided then recollected one year later to record the difference. Finally, the greatest limitation to this study in part is the anonymity of the shipping company, as it limits the amount of evidence that the authors can provide. Conflicts of interest declaration: The authors have no conflict of interest to declare.

\section{Conclusion}

Based on the subjective data collected via survey and the decrease in OSHA reported work-related injuries the authors conclude that with proper education and awareness regarding stretching, exercise, and proper biomechanics it is possible to decrease the number of work-related injuries within the shipping and receiving industry.

\section{Appreciation}

The primary researchers would like to thank Dr. Carley for his guidance and encouragement to pursue this study to add to the body of knowledge and the need for assessment in the field of ergonomic training.

\section{References}

1. Shuai J, Yue P, Li L, Liu F, Wang S (2014) Assessing the effects of an educational program for the prevention of work-related musculoskeletal disorders among school teachers. BMC Public Health 14(1): 1211.

2. Ranasinghe P, Perera YS, Lamabadusuriya DA, Kulatunga S, Jayawardana N, et al. (2011) Work related complaints of neck, shoulder and arm among computer office workers: a cross-sectional evaluation of prevalence and risk factors in a developing country. Environmental Health 10(1): 70.

3. Zebis $\mathrm{M}$, Andersen L, Pedersen M, Peter M, Andersen $\mathrm{CH}$, et al. (2011) Implementation of neck/shoulder exercises for pain relief among industrial workers: A randomized controlled trial. BMC Musculoskeletal Disorders 12(1): 205.

4. Juul-Kristensen B (2005) Self-reported workplace related ergonomic conditions as prognostic factors for musculoskeletal symptoms: the "BIT" follow up study on office workers. Occupational and Environmental Medicine 62(3): 188-194.

5. Habibi E, Soury S (2015) The effect of three ergonomics interventions on body posture and musculoskeletal disorders among stuff of Isfahan Province Gas Company. Journal of Education and Health Promotion 4(1): 65.

6. Kincl L, Anton D, Hess J, Weeks D (2016) Safety voice for ergonomics (SAVE) project: protocol for a workplace cluster-randomized controlled trial to reduce musculoskeletal disorders in masonry apprentices. BMC Public Health 16(1): 362.

7. Driessen M, Proper K, Anema J, Bongers $\mathrm{P}$, van der Beek A (2010) Process evaluation of a participatory ergonomics programme to prevent low back pain and neck pain among workers. Implementation Science 5(1): 65. 